\title{
Impacto da pandemia COVID-19 no tratamento de pacientes oncológicos e suas consequências psicológicas
}

\author{
Impact of the COVID-19 pandemic on the treatment of cancer patients and its \\ psychological consequences
}
Impacto de la pandemia de COVID-19 en el tratamiento de pacientes con cáncer y sus consecuencias psicológicas

Tífanny Beatriz Brito Mendes ${ }^{1 *}$, João Victor Santana Araújo ${ }^{1}$, Thâmara Almeida Botelho1, Maria Rafaela Alves Nascimento², lury Marcos da Silva Pessoa², Gustavo Santos Viana ${ }^{2}$, Yure Batista de Sousa $^{2}$, Rinara Soares de Oliveira ${ }^{1}$, Pedro Câmara de Assis ${ }^{1}$, Fillipe Dantas Pinheiro.

\section{RESUMO}

Objetivo: Compreender os principais aspectos relacionados a interrupção dos tratamentos oncológicos durante a pandemia de COVID-19 e os aspectos psicológicos envolvidos. Revisão bibliográfica: A pandemia COVID-19 impactou diretamente no tratamento de pacientes oncológicos. Além disso, esses pacientes possuem maior probabilidade do que a população geral de serem infectados, ou de desenvolverem fenótipos graves do COVID-19 devido seu estado imunodeprimido, somado a visitas frequentes aos hospitais como ponto de infecção e das terapias imunossupressoras para o tratamento do câncer, além de fatores de risco adicionais a depender da idade e tipo de câncer. Ademais, os paciente oncológicos tem níveis aumentados de estresse percebido e pós-traumático atenuado com a quarentena e o contexto vigente de instabilidades. Sendo evidenciado a coronofobia como causa da elevação dos níveis de depressão, ansiedade generalizada e tanafobia em comparação aos anteriores a pandemia. Considerações finais: Nesta perspectiva, torna-se necessário maiores pesquisas sobre o impacto da pandemia no tratamento de pacientes oncológicos, sobre a interação do câncer com o COVID-19 e fornecimento de suporte psicossocial a essa população.

Palavras-chave: COVID-19, SARS-CoV-2, Pandemia, Câncer, Pacientes oncológicos.

\begin{abstract}
Objective: To understand the main aspects related to the interruption of cancer treatments during the COVID19 pandemic and the psychological aspects involved. Bibliographic review: The COVID-19 pandemic had a direct impact on the treatment of cancer patients. In addition, these patients are more likely than the general population to be infected, or to develop severe COVID-19 phenotypes due to their immunocompromised status, plus frequent visits to hospitals as a point of infection and immunosuppressive therapies for cancer treatment, plus additional risk factors depending on age and type of cancer. Furthermore, cancer patients have increased levels of perceived and post-traumatic stress, attenuated by quarantine and the current context of instabilities. Coronophobia being evidenced as a cause of increased levels of depression, generalized anxiety and tanaphobia compared to previous pandemics. Final considerations: From this perspective, further research is needed on the impact of the pandemic on the treatment of cancer patients, on the interaction of cancer with COVID-19 and the provision of psychosocial support to this population.
\end{abstract}

Keywords: COVID-19, SARS-CoV-2, Pandemic, Cancer, Cancer patients.

${ }^{1}$ Faculdade Santo Agostinho (FASA), Vitória da Conquista - BA. *E-mail: beatriztifanny2@gmail.com

2 Centro Universitário FIPMoc (UNIFIPMoc), Montes Claros - MG.

SUBMETIDO EM: 11/2021 


\section{RESUMEN}

Objetivo: Comprender los principales aspectos relacionados con la interrupción de los tratamientos oncológicos durante la pandemia de COVID-19 y los aspectos psicológicos involucrados. Revisión bibliográfica: La pandemia de COVID-19 tuvo un impacto directo en el tratamiento de los pacientes con cáncer. Además, estos pacientes tienen más probabilidades que la población general de estar infectados o de desarrollar fenotipos graves de COVID-19 debido a su estado de inmunodepresión, además de visitas frecuentes a hospitales como punto de infección y terapias inmunosupresoras para el tratamiento del cáncer, más riesgo adicional. factores que dependen de la edad y el tipo de cáncer. Además, los pacientes con cáncer tienen niveles aumentados de estrés percibido y postraumático, atenuado por la cuarentena y el contexto actual de inestabilidad. La coronofobia se evidencia como una causa de aumento de los niveles de depresión, ansiedad generalizada y taanofobia en comparación con pandemias anteriores. Consideraciones finales: Desde esta perspectiva, se necesitan más investigaciones sobre el impacto de la pandemia en el tratamiento de pacientes con cáncer, sobre la interacción del cáncer con COVID-19 y la provisión de apoyo psicosocial a esta población.

Palabras clave: COVID-19, SARS-CoV-2, Pandemia, Cáncer, Pacientes con cáncer.

\section{INTRODUÇÃO}

O COVID-19 se associa com fenótipos variados, com apresentações que variam de infecções assintomáticas e quadros leves à severos, com pneumonia viral grave, síndrome do desconforto respiratório agudo e morte (HANNA TP, et al., 2020; LEE LY, et al., 2020b). Desde os seus primeiros casos descritos em 2020, que foram relatados na China no início de janeiro e se espalhou posteriormente para os Estados Unidos e quase 200 países, a comunidade oncológica teve que se adaptar a uma emergência médica em constante evolução enquanto tentava garantir a saúde e segurança aos pacientes com câncer (PRINTZ C, 2020).

Apesar dos esforços realizados, a pandemia impactou diretamente o tratamento de câncer dos pacientes oncológicos, incluindo encurtamento de radioterapia, mudanças nos esquemas de quimioterapia intravenosa para oral e modificações no uso de imunoterapias ocorreu ainda o adiamento de diagnósticos, devido a carga sem precedentes do COVID-19 nos sistemas de saúde (LEE LY, et al., 2020a; PAPAUTSKY EL e HAMLISH T, 2020).

Segundo o Instituto Nacional de Câncer (INCA) o Brasil registra 625 mil novos casos de câncer para cada ano do triênio 2020/2022, ainda estima uma incidência de 309.750 novos casos de Câncer (CA) em homens no Brasil em 2020, sendo as localizações primárias mais incidentes de próstata (29,2\%), Colorretal $(9,1 \%)$ e de Traqueia, Brônquio e pulmão (7,9\%). Já nas mulheres estima-se o surgimento de 316.280 novos casos no mesmo ano, sendo o CA de mama (29,7\%), Cólon e reto $(9,2 \%)$ e Colo do útero $(7,5 \%)$, os com maiores incidências respectivamente. Nos Estados Unidos, mais de 16 milhões de pessoas atualmente tem ou sobreviveram ao câncer (cerca de $5 \%$ da população), sendo portanto, inevitável que muitos deles sejam afetados pelo COVID-19 (SHAH UA, 2020).

Além disso, estudos demostram que os pacientes com câncer têm maior probabilidade do que a população geral de serem infectados e de sofrerem doenças graves que levam ao óbito, havendo susceptibilidades diferentes a depender do tipo de tumor, sendo observado um aumento notável de apresentações hospitalares em pacientes com CA hematológicos (LEE LY, et al., 2020b). Dessa forma, essa população deve seguir rigorosamente o isolamento social, que, aliado com aos aspectos emocionais próprios do câncer, agrava as repercussões psicológicas desses pacientes, gerando uma grande preocupação em oncologistas e pacientes sobre quais medidas devem ser tomadas (SHAH UA, 2020).

Para a contenção do estágio inicial da crise COVID-19 vem sendo utilizada o distanciamento social restritivo, sendo sua estratégia a redução dos números de casos graves no sistema de saúde, de forma a diminuir o número de mortes evitáveis por sobrecarga de recursos. Além disso, os pacientes com câncer têm alto risco de desenvolver a forma grave do COVID-19, dessa forma as Diretrizes vêm propondo ajustes nos tratamentos oncológicos (KUTIKOV A, et al., 2020). 
Nesse contexto, o isolamento social com o fim de reduzir potenciais oportunidades de transmissão viral, leva a uma interrupção de acesso a vários tratamentos oncológicos que desencadeia um efeito cascata, com suspensão de visitas clinicas, estadias cirúrgicas, sessões de quimioterapia, planejamento de radiação, internações, realização de exames laboratoriais e de imagem, entre outros. A entrepausa e modificações de tratamento exigem uma avaliação adicional para determinar as consequências clínicas, se houver, que ocorreu para esses pacientes (DISIS ML, 2020).

Outro aspecto a ser observado, é que os pacientes em terapia ativa durante a pandemia, bem como prestadores de cuidados oncológicos, têm níveis aumentados de estresse percebido e pós-traumático, sendo necessária uma análise mais detalhada desse contexto e das lacunas nos recursos de suporte psicossocial que ajudam a promover a resiliência emocional e a reduzir o risco de estresse pós-traumático em pacientes em terapia ativa (JOLY F, et al., 2020).

A experiência de quarentena, autoisolamento, bloqueio e crise econômica podem atuar como carga alostática para a população geneticamente vulnerável com predisposição a doenças de saúde mental. As ramificações psiquiátricas podem variar de problemas com enfrentamento e exacerbação ou recaída de diagnósticos psiquiátricos subjacentes. Além disso, a infecção viral pode estar diretamente no centro da etiopatogênese das síndromes psiquiátricas como foi demonstrado na esquizofrenia (NAGUY A, et al., 2020).

Diante deste cenário descrito, mostra-se relevante essa revisão narrativa, que objetiva compreender os principais aspectos relacionados a interrupção dos tratamentos oncológicos durante a pandemia de COVID19 e os aspectos psicológicos envolvidos, contribuindo com informações para o desenvolvimento de estratégias de intervenções institucionais para a proteção da saúde e bem-estar dessa população.

\section{REVISÃO BIBLIOGRÁFICA}

\section{Interações entre o covid-19 e o câncer}

Os pacientes com câncer são mais vulneráveis a resultados desfavoráveis da infecção por coronavírus, incluindo a maior necessidade de suporte ventilatório (RICHARDS M, 2020). Em análise de pesquisas realizadas no Hospital Zhongnam da Universidade de Wuhan, na China, o paciente com câncer tem duas vezes mais chances de serem infectados pelo COVID-19, outra pesquisa relata uma incidência de COVID-19 que varia de 0,5 a $6 \%$, com uma mortalidade de aproximadamente $25,0 \%$ em pacientes com câncer (AMERICAN ASSOCIATION FOR CANCER RESEARCH, 2020; LIU Y, et al., 2021).

As primeiras estimativas da China sugerem uma taxa geral de letalidade de pacientes oncológicos que adquirem o COVID-19 de $2 \%$, aumentando para $8 \%$ para pessoas de $70-79$ anos de idade e $15 \%$ para aqueles com $\geq 80$ anos de idade. As taxas de letalidade também são marcadamente mais altas entre pacientes com comorbidades: $11 \%$ para doenças cardiovasculares, $7 \%$ para diabetes, $6 \%$ para doenças respiratórias crônicas e 6\% para câncer (HANNA TP, et al., 2020). Além disso, os pacientes com câncer são frequentemente mais velhos (ou seja, com idade $>60$ anos) aumentando ainda mais os riscos relacionados ao COVID-19 (KUDERER NM, et al., 2020). No entanto, ainda existem dificuldades para determinar se o COVID-19 se trata da causa direta da morte do paciente, ou se é ocasionada por um evento terminal devido ao câncer (LEE LY, et al., 2020b).

Quando se trata dos pacientes oncológicos pediátricos, parece ser visto um cenário diferente, onde a maioria, quando infectados pelo SARS-CoV-2, tem quadros de infecções leves ou assintomáticas, onde $87 \%$ têm febre e $62 \%$ têm tosse ou sintomas de coriza na apresentação, a maioria não necessita de tratamento hospitalar especificamente pela causa do COVID-19 (85\%), sugerindo que esse grupo de pacientes não apresentam alto risco (MILLEN GC, et al., 2020).

Além disso, paciente com diferentes tipos de tumor possuem susceptibilidades diferentes a doença ao SARS-CoV-2 e diferentes fenótipos da doença COVID-19, sendo notável um aumento das apresentações graves em pacientes com neoplasias hematológicas (leucemia, linfoma e mieloma), exigindo intervenções de suporte mais intensivo em comparação as outras neoplasias. Por outro lado, é observado um risco menor de contração e mortalidade em pacientes com CA de mama e doenças malignas do trato genital feminino (LEE LY, et al., 2020a). 
Várias razões teóricas podem ajudar no entendimento dessa maior vulnerabilidade dos pacientes oncológicos adultos, dentre elas temos: a quimioterapia, que levaria a uma neutropenia e linfopenia, potencialmente predispondo o paciente a uma maior chance de infecção; as terapias com inibidores do ponto de controle imunológico, tratamento comum para melanoma e câncer de pulmão, que podem em alguns pacientes aumentar o risco de síndromes hiperinflamatórias e doença respiratória aguda; e a radioterapia que pode limitar a reserva da medula óssea e pode ocasionar pneumonite, apesar de serem manifestações raras e associadas ao uso de aparelhos antigos, podem aumentar o risco de complicações relacionadas ao COVID19 (DISIS ML, 2020; LIANG W, et al., 2020).

A interrupção imunológica observada em pacientes com leucemia e o uso de tratamentos com mielossupressores também podem resultar em combinação de riscos, com aumento da probabilidade da infecção inicial por SAR-CoV-2, sua capacidade de ser instalar no hospedeiro e probabilidade de desenvolvimento de fenótipos graves, com a tempestade de citocinas e falência de múltiplos órgãos (LEE LY, et al., 2020b).

Pesquisas mais recentes observaram uma interação recíproca do câncer com o COVID-19. A princípio descobriu-se que a glicoproteína de Spike (S) medeia a entrada celular do SARS-CoV-2 via ligação à enzima conversora de angiotensina 2 (ACE2) após proteólise pela protease transmembrana serina 2 (TMPRSS2), clivando além da ACE2 a proteína do SARS-CoV-2, participando da fusão da membrana e da captação celular do vírus. De maneira oposta a proteína 17 contendo o domínio de desintegrina e metaloproteinase (ADAM17) induz a liberação da proteína clivada de ACE2 no espaço celular extracelular. Estudos promissores apontam a possibilidade do uso de proteínas ACE2 modificadas molecularmente como ferramenta terapêutica potencial no combate de disseminação da SARS-CoV-2. Consequentemente, quando existe uma alta carga viral, a barreira de eliminação viral mediado por ADAM17-ACE2 é superado, facilitando a infecção subsequente (BREST P, et al., 2020).

Alguns tratamentos específicos do câncer causam repercussões nesses fatores-chaves intrínsecos citados anteriormente (ADAM17 e TMPRSS2). O TMPRSS2 desempenha importante papel no câncer de próstata, já que ele tem sua expressão gênica fortemente regulada por andrógenos. Sendo assim, com o tratamento antiandrógeno amplamente usado pode ocorrer uma diminuição da expressão das células TMPRSS2, levando uma vulnerabilidade desses pacientes (geralmente de faixa etária mais elevada, pela epidemiologia desse câncer) para a infecção pelo COVID-19. Já os tratamentos realizados pelos inibidores de tirosina quinase (TKI) anti-EGFR aumentaria a susceptibilidade de COVID-19 por meio da redução do ADAM17 (MONTOPOLI $M$, et al., 2020).

Temos ainda, o uso da quimioterapia que pode aumentar a susceptibilidade da infecção, a exemplo do inibidor do ponto de verificação imunológico (ICI) que leva a uma liberação de citocinas (tempestade inflamatória) mediadas por ICI representando um problema clínico na fase inicial do COVID-19 agravando a morbidade induzida e complicando o curso do quadro, já que ocorre uma hiperativação de células $T$ devido uma desregulação imunológica, podendo gerar uma exacerbação da lesão pulmonar, favorecendo a síndrome do desconforto respiratório. Porém, ainda não está claro este efeito imunológico e o real impacto da infecção por COVID-19, devendo ser realizados mais pesquisas nesse âmbito (BREST P, et al., 2020; LIU Y, et al., 2021).

A infecciosidade de COVID-19 pode ser agravada por um aumento em TMPRSS2 sob a presença de terapia anti-androgênica ou por uma diminuição mediada por vandetanibe na expressão de ADAM17. Por outro lado, a quimioterapia imunossupressora e os inibidores do ponto de verificação imunológico podem contribuir para o agravamento dos sintomas relacionados ao COVID-19 (BREST P, et al., 2020).

Outras características clínicas além das já citadas parecem ter um risco significativamente aumentado de incidência e mortalidade pelo COVID-19, como paciente idoso, sexo masculino, etnia, comorbidades associadas (hipertensão, doença isquêmica do coração, diabetes, doença pulmonar crônica), uso de esteroides e tempo de tratamento antitumoral (LIU Y, et al., 2021).

Os achados imunopatológicos do COVID-19 são caracterizados pela apoptose de células T sensibilizadas, com adicional atraso na produção de interferons (IFN). Na fase inicial da infecção há aumento da expressão 
de morte celular programada-1 (PD-1) e na morte celular programada-ligante-1 (PD-L1), à medida que ocorre a progressão da doença há menor imunidade humoral e celular contra infecções virais devido ao comprometimento funcional dos linfócitos T CD4+ e a exaustão dos linfócitos T citotóxicos CD8+ (LIU Y, et al., 2021).

Além dessas questões imunológicas citadas, o próprio ambiente de tratamento, acompanhamento e constante realização de procedimentos em pacientes oncológicos aumentam as chances de contrair o COVID-19 (YU J, et al., 2020). Diversas vias de tratamento foram alteradas na tentativa de minimizar a exposição potencial de pacientes com câncer ao SARS-CoV-2 e para reduzir o risco durante a cirurgia ou terapia de radiação (RICHARDS M, 2020).

Cada paciente que está inserido no sistema de prestação de cuidados oncológicos interrompe significativamente a tática de distanciamento social e resulta em um grande número de pontos de contato pessoal e assim oportunidades potenciais para a transmissão viral (KUTIKOV A, et al., 2020).

Assim, oncologistas e seus pacientes tiveram que tomar decisões difíceis com o objetivo de limitar a exposição potencial ao vírus COVID-19, influenciando nas escolhas de tratamento. Esses profissionais precisam pesar os riscos de morte e morbidade do COVID-19 contra a magnitude do benefício das terapias contra o câncer pretendidas (DISIS ML, 2020).

Liang W, et al. (2020) propõe três estratégias principais para pacientes com câncer durante a crise do COVID-19. Primeiro, deve ser feito um adiamento intencional da quimioterapia adjuvante ou cirurgia eletiva para pacientes com quadro estável inserido em regiões endêmicas. Na tentativa de auxiliar nessa tomada de decisões, uma equipe de cientistas e médicos do Michigan Public Health e da University of Michigan Rogel Cancer Center desenvolveram um aplicativo gratuito que ajuda os médicos a comparar o risco de longo prazo para pacientes com câncer, o OncCOVID App, se mostrando promissor na alocação de recursos de tratamento individualizado para pacientes com câncer durante a pandemia global COVID-19 (HARTMAN HE, et al., 2020).

Em segundo lugar, deve ser realizado um reforço das medidas de proteção pessoal dessa população (LIANG W, et al.,2020). Levando em consideração a definição da UNESCO do princípio da precaução, na qual é tratada de atividades que apesar de serem plausíveis cientificamente são moralmente inaceitáveis, devendo-se nesses casos adotar medidas para redução de danos, assim outros cuidados devem ser tomados na abordagem desses pacientes, como, redução dos tempos de espera para realização dos procedimentos em ambiente hospitalar, sendo mantidos tão curtos quanto razoavelmente possível (HANNA TP, et al., 2020).

E em terceiro, a vigilância e o tratamento mais intensivo devem ser considerados quando pacientes com câncer são infectados com Sars-CoV-2, especialmente aqueles de maiores faixas etárias e com presença de outras comorbidades (LIANG W, et al., 2020).

\section{Impactos nos tratamentos oncológicos}

Segundo Papautsky EL e Hamlish T (2020), nas primeiras semanas da pandemia de COVID-19, quase metade de todos os sobreviventes do câncer de mama sofreram atrasos no seu tratamento oncológico. Além disso, ele afirma que houve uma priorização das Diretrizes dos EUA focadas no gerenciamento do COVID19, ao invés das necessidades de saúde de paciente com câncer de mama que requerem acesso a serviços essenciais.

Todavia ainda existe uma tentativa das diretrizes emergentes em equilibrar os riscos de atrasos no tratamento de câncer e o aumento da demanda por recursos médicos devido ao contexto de pandemia atual (PAPAUTSKY EL e HAMLISH T, 2020).

A interrupção do tratamento oncológico normal em virtude da pandemia ocorreu por vários motivos. Primeiro, os oncologistas e o restante da equipe de câncer estão sob pressões sem precedentes. Essas pressões incluem preocupação crescente dos pacientes sobre sua vulnerabilidade percebida, operações de câncer canceladas, um impulso substancial para fazer telemedicina em vez de consultas presenciais e um alto grau de ausência do trabalho na equipe de câncer devido a doenças pessoais e auto isolamento. Em 
segundo lugar, muitos oncologistas estão sendo redistribuídos para funções de medicina geral ou aguda para apoiar as muitas admissões de COVID-19 que requerem suporte médico intensivo e informações (LEE LY, et al., 2020a).

É relatado que ocorreram modificações no manejo do câncer em $27 \%$ dos pacientes, sendo que dentre esses, $15 \%$ tiveram interrupção do tratamento e 19\% tiveram mudanças no esquema de administração. Notavelmente, as mudanças no esquema de administração foram mais comuns em pacientes que iniciaram o tratamento durante o bloqueio lockdown pela pandemia (33,0\%) (JOLY F, et al, 2020).

Observando o cenário mundial ocorreram também impactos significativos em outros países na prestação de cuidados contra o câncer, a exemplo da terapia anticâncer sistêmica (SACT) no Reino Unido, onde ocorreu um decréscimo de $28,7 \%$ dos atendimentos, sendo o impacto maior nos tratamentos de câncer colorretal, e menor no câncer de mama (BAXTER MA, et al., 2021).

Ainda no contexto do Reino Unido, mais especificamente para o câncer de olho, nos Serviços de Oncologia Ocular foram observados uma redução considerável nos números de tratamentos, encaminhamentos e de novos casos diagnosticados de melanoma uveal no período de 4 meses do bloqueio devido a pandemia (WANG H, et al., 2020).

O impacto do tratamento tardio do câncer em comparação com o imediato varia substancialmente dentro dos tipos e estágios do câncer. Por exemplos, pacientes com CA de próstata têm impacto mínimo no tratamento retardado, já que são pacientes que passam apenas 1 a 2 dias no hospital para procedimento cirúrgico ou recebem tratamentos ambulatoriais breves com radioterapia, diferentemente do que ocorre entre os pacientes com câncer pancreático, que precisam de uma permanência hospitalar maior (HARTMAN HE, 2020).

Outro ponto que deve ser destacado é que os pacientes com câncer parecem ter maior risco de mortalidade e doença grave devido à infecção por SARS-CoV-2, independentemente de terem câncer ativo, estarem em tratamento anticâncer ou ambos (KUDERER NM, et al., 2020)

\section{Impactos emocionais da pandemia em pacientes oncológicos}

Pesquisas realizadas sobre o COVID-19 e os relatórios da mídia revelaram um aumento nos temores relacionados a contrair o vírus. Embora o medo seja um resultado psicológico comum durante as pandemias, essa especificamente trata-se de um surto patológico em evolução contínua e tem fatores de risco únicos. Portanto, o sentimento apresentado nesse contexto pode se manifestar não apenas em medo e ansiedade relacionados à contração da doença e à morte, mas também ao estresse sócio-ocupacional associado (ALISHA A, et al., 2020).

Um novo construto denominado Coronafobia vem sendo utilizado para descrever essa nova fobia emergente específica do COVID-19 e atualmente é uma importante variável da saúde mental, estando fortemente relacionado ao comprometimento funcional e sofrimento psicológico (SHERMAN AL, et al., 2020).

A patogênese dessa condição se relaciona com fatores de riscos como: sentimento de inserção em uma realidade imprevista; incertezas sem fim; necessidade de aquisição de novas práticas e comportamento de evitação (autoisolamento); perda de fé na infraestrutura de saúde; declarações de advertência de organismos internacionais; e infodemia - um excesso de informações, algumas precisas e outras não, que tornam difícil encontrar fontes idôneas e orientações confiáveis quando se precisa (GARCIA LP e DUARTE E, 2020). Supõe-se que esses fatores causam interferência no cotidiano dos indivíduos, levando a uma interpretação catastrófica dos acontecimentos mundiais que levam à coronafobia (ALISHA A, et al., 2020).

Segundo pesquisas recentes, foi evidenciado que essa fobia explica a variação adicional na depressão e transtorno de ansiedade generalizada, acima dos níveis anteriores a pandemia. Podendo assumir formas de ataques de pânico, tanatofobia (medo excessivo da morte), hipocondria, surtos de TOC relacionados à contaminação com ablutomania (lavagem compulsiva ou estocagem excessiva de mantimentos), entre outros (NAGUY A, et al., 2020). Sendo assim, de extrema importância que os profissionais de saúde estejam cientes da coronafobia (SHERMAN AL, et al., 2020). 
Segundo Joly F, et al (2020), os pacientes com terapia oncológica ativa, bem como prestadores de cuidados oncológicos, apresentam níveis aumentados de estresse percebido e pós-traumático durante a pandemia de COVID-19, demonstrado nos estudos preliminares do COVIPACT. Percebe-se assim uma lacuna existente no suporte psicossocial, na promoção da resiliência e redução do estresse e ansiedade desses indivíduos, tendo a psiquiatria e a psicologia papeis fundamentais nessa crise (NAGUY A, et al., 2020).

Os efeitos psicológicos adversos do COVID-19 aumentaram globalmente, em junção a isso, o custo psicológico pode estar piorando para esta crise devido aos números crescentes de mortes e níveis de desemprego (SHERMAN AL, et al., 2020).

\section{CONSIDERAÇÕES FINAIS}

Diante dos dados apresentados é possível concluir a existência da vulnerabilidade dos pacientes oncológicos no contexto vigente da pandemia, com maior exposição aos fatores de risco. Nesta perspectiva, é de extrema necessidade que sejam realizadas maiores pesquisas para basear as tomadas de decisões na continuidade e adequação dos tratamentos vigentes de pacientes oncológicos, fundamentado em protocolos visando a redução de riscos. Além disso, diante dos impactos psicológicos desses pacientes e de seus cuidadores, é fundamental a disponibilidade de suporte psicossocial, reduzindo os danos psicológicos e psiquiátricos da pandemia, especialmente nessa população que possui carga adicional de fragilidade emocional pela condição oncológica.

\section{REFERÊNCIAS}

1. ALISHA A, et al. Understanding coronaphobia. Asian Journal of Psychiatry, 2020; 54.

2. AMERICAN ASSOCIATION FOR CANCER RESEARCH. COVID-19 More Frequent, Severe in Cancer Patients. Cancer Discovery, 2020; 10(6).

3. BAXTER MA, et al. The impact of COVID-19 on systemic anticancer treatment delivery in Scotland. Br J Cancer, 2021; 124: 1353-1356.

4. BREST P, et al. More light on cancer and COVID-19 reciprocal interaction. Br J Cancer, 2021; 124: 1344-1345.

5. DISIS ML. Oncologia e COVID-19. JAMA, 2020.

6. GARCIA LP, DUARTE E. Infodemia: excesso de quantidade em detrimento da qualidade das informações sobre a COVID19. Epidemiol. Serv. Saúde, 2020.

7. HANNA TP, et al. Cancer, COVID-19 and the precautionary principle: prioritizing treatment during a global pandemic. Nature reviews. Clinical oncology, 2020; 17.

8. HARTMAN HE, et al. Integrated Survival Estimates for Cancer Treatment Delay Among Adults With Cancer During the COVID19 Pandemic. JAMA Oncol, 2020; 6: 1881-1889.

9. INSTITUTO NACIONAL DE CÂNCER (INCA). Estatísticas de câncer. 2021. Disponível em: https://www.inca.gov.br/numerosde-cancer. Acessado em: 23 de Abril de 2021.

10. JOLY F, et al. LBA69: impact of the COVID-19 pandemic on management of medical cancer treatments and psychological consequence for the patients. Ann Oncol, 2020.

11. KUDERER NM, et al. Clinical impact of COVID-19 on patients with cancer (CCC19): a cohort study. Lancet. London, 2020; 395: 1907-1918.

12. KUTIKOV A, et al. A War on Two Fronts: Cancer Care in the Time of COVID-19. Annals of internal medicine, $2020 ; 172$.

13. LEE LY, et al. COVID-19 mortality in patients with cancer on chemotherapy or other anticancer treatments: a prospective cohort study. Lancet. London, 2020a; 395: 1919-1926.

14. LEE LY, et al. COVID-19 prevalence and mortality in patients with cancer and the effect of primary tumour subtype and patient demographics: a prospective cohort study. Lancet. London, 2020b; 21(10): 1309-1316.

15. LIANG W, et al. Cancer patients in SARS-CoV-2 infection: a Nationwide analysis in China. The Lancet Oncology, $2020 ; 21$.

16. LIU Y, et al. Does prior exposure to immune checkpoint inhibitors treatment affect incidence and mortality of COVID-19 among the cancer patients: The systematic review and meta-analysis. International Immunopharmacology, 2021; 101: Part A.

17. MILLEN GC, et al. Severity of COVID-19 in children with cancer: Report from the United Kingdom Paediatric Coronavirus Cancer Monitoring Project. Br J Cancer, 2021; 124: 754-759.

18. MONTOPOLI M, et al. Androgen-deprivation therapies for prostate cancer and risk of infection by SARS-CoV-2: a populationbased study $(N=4532)$. Ann. Oncol, 2020; 31: 1040-1045.

19. NAGUY A, et al. Coronaphobia and chronophobia - A psychiatric perspective. Asian journal of psychiatry, $2020 ; 51$.

20. PAPAUTSKY EL, HAMLISH T. Patient-reported treatment delays in breast cancer care during the COVID-19 pandemic. Breast Cancer Res Treat, 2020.

21. PRINTZ C. When a global pandemic complicates cancer care: Although oncologists and their patients are accustomed to fighting tough battles against a lethal disease, Coronavirus Disease 2019 (COVID-19) has posed an unprecedented challenge. Cancer, 2020; 126: 3171 .

22. RICHARDS M, et al. The impact of the COVID-19 pandemic on cancer care. Nature cancer, 2020.

23. SHAH UA. Cancer and Coronavirus Disease 2019 (COVID-19) - Facing the "C Words". JAMA Oncol, 2020.

24. SHERMAN AL, et al. Incremental validity of coronaphobia: Coronavirus anxiety explains depression, generalized anxiety, and death anxiety. Journal of Anxiety Disorders, 2020; 74.

25. WANG H, et al. Impact of COVID-19 pandemic on eye cancer care in United Kingdom. Br J Cancer, 2021; 124: 1357-1360.

26. YU J, et al. SARS-CoV-2 Transmission in Patients With Cancer at a Tertiary Care Hospital in Wuhan, China. JAMA Oncol, 2020. 